\title{
NULL SYSTEMS IN PROJECTIVE SPACE
}

\section{REINHOLD BAER}

If $P$ is an (abstract) $n$-dimensional projective space, then we define a polarity in $P$ as a correspondence $p$ associating with every point $Q$ in $P$ a hyperplane $Q^{p}$ and with every hyperplane $h$ in $P$ a point $h^{p}$ in such a way that:

(i) $Q=Q^{p^{2}}$ for every point $Q$ and $h=h^{p^{2}}$ for every hyperplane $h$.

(ii) The point $Q$ is on the hyperplane $h$ if, and only if, the hyperplane $Q^{p}$ passes through the point $h^{p}$.

It is an immediate consequence of (i) that polarities are 1:1 correspondences.

We shall term $p$ a null-polarity if the polarity $p$ has the additional property that:

(iii) Every point $Q$ is on the corresponding hyperplane $Q^{p}$, and consequently every hyperplane $h$ passes through the corresponding point $h^{p}$.

Extending a result of Veblen and Young, R. Brauer ${ }^{1}$ has shown that the existence of a null-polarity in $P$ implies that the number $n$ of dimensions of $P$ is odd, and he has connected the null-polarities with the so-called null-systems, provided $P$ is the $n$-dimensional projective space over a commutative field of coordinates. It is the object of the present note to show that this last hypothesis may be omitted; more precisely we are going to show that if the dimension of $P$ is greater than 1 , then the existence of a null polarity is equivalent to the fact that $P$ is of odd dimension and is a projective space over a commutative field of coordinates.

If $P$ is a projective space of dimension 1 , then the hyperplanes are points too. The identity transformation on the points of the line $P$ is therefore the null-polarity of $P$. For this reason we shall assume throughout the remainder of this note that $P$ be of dimension greater than 1.

The case of a projective plane $P$ has to be treated separately from the others, since the Theorem of Desargues need not hold true in a projective plane, though it is true for all the higher-dimensional projective spaces.

A projective plane is a system of points and lines such that any two different lines meet in one and only one point, any two different

Presented to the Society, September 17, 1945; received by the editors May 31, 1945.

${ }^{1} \mathrm{R}$. Brauer, A characterization of null systems in projective space, Bull. Amer. Math. Soc. vol. 42 (1936) pp. 247-254. 
points determine one and only one line (on which they are both situated) and such that every line carries at least three points (every point is on at least three lines).

LEMma. If $p$ is a polarity of the projective plane $P$, if $Q$ and $R$ are different points in $P$ such that $Q$ is on the line $Q^{p}$ and $R$ is on the line $R^{p}$, then $Q$ is not on $R^{p}$ (nor is $R$ on $Q^{p}$ ).

Proof. If $Q$ were on $R^{p}$, then $R^{p}$ would be the uniquely determined line through the two different points $R$ and $Q$, since $R$ is on $R^{p}$. Furthermore $R$ would be on $Q^{p}$; and it would follow likewise that $Q^{p}$ is the uniquely determined line through $R$ and $Q$. Thus $R^{p}=Q^{p}$ and hence $R=Q$, a contradiction proving our contention.

COROLLARY. There does not exist a null-polarity in a projective plane.

This is an immediate consequence of the lemma.

Because of the corollary we shall assume throughout the remainder of this note that the dimension of the projective space $P$ is at least 3 . In this case $P$ is the $n$-dimensional projective space over an essentially uniquely determined, not necessarily commutative, field $F$.

If $F$ is any field (commutative or not), and if $n$ is an integer not less than 3 , then we denote by $(F, n)$ an additively written abelian group, admitting the elements in $F$ as left-multipliers, and having the rank $n+1$ over $F$. The $n$-dimensional projective space over $F$ is then essentially the same as the partially ordered set $P(F, n)$ of all the $F$-admissible subgroups of $(F, n)$, the points being of the form $F x$ with $x \neq 0$, and the hyperplanes being of rank $n$.

Every polarity $p$ of $P(F, n)$ may, as is well known, ${ }^{2}$ be represented in the following form: There exist an anti-automorphism $a$ of $F$ (satisfying $\left.a^{2}=1\right)$ and an $F$-valued function $f(x, y)$, for $x, y$ in $(F, n)$, satisfying: $:^{3}$

$f(x, y)=0$ if, and only if, $f(y, x)=0$;

$f(u x+v y, z)=u f(x, z)+v f(y, z), f(z, u x+v y)=f(z, x) u^{a}+f(z, y) v^{a}$.

The point $F x$ is on the hyperplane $(F y)^{p}$ if, and only if, $f(x, y)=0$.

Assume now that the polarity $p$ be a null-polarity. This is equivalent to saying $f(x, x)=0$ for every $x$ in $(F, n)$. If $x \neq 0$, then there exists

${ }^{2} \mathrm{~A}$ proof of this fact may be effected in essentially the same fashion as done by R. Brauer, op. cit. pp. 251, 252, in the case of commutative $F$; for a detailed proof of a more comprehensive fact see R. Baer, $A$ unified theory of projective spaces and finite abelian groups, Trans. Amer. Math. Soc. vol. 52 (1942) pp. 315-317.

${ }^{3}$ We state here only such properties of the function $f(x, y)$ as will be needed later. Further properties have to be imposed to assure that, conversely, every such $f(x, y)$ defines a polarity. Note in particular that no use has been made of the involutorial character of $a$. 
at least one $y$ such that $f(x, y) \neq 0$, since otherwise $(F x)^{p}$ would be the whole space instead of only a hyperplane. Let $x, y$ be any pair of elements such that $f(x, y) \neq 0$. If $t$ is some element in $F$, then we find

$$
\begin{aligned}
0 & =f(x+t y, x+t y)=f(x, x)+f(x, y) t^{a}+t f(y, x)+t f(y, y) t^{a} \\
& =f(x, y) t^{a}+t f(y, x) .
\end{aligned}
$$

Substituting $t=1$, we obtain $f(x, y)+f(y, x)=0$; and thus the above equation reduces to $0=f(x, y) t^{a}-t f(x, y)$. Since $f(x, y) \neq 0$, this implies $t^{a}=f(x, y)^{-1} t f(x, y)$ for every $t$ in $F$, proving that the anti-automorphism $a$ of $F$ is an inner automorphism of $F$. Hence $F$ is commutative and $a=1$. Combining this with the fact that $f(x, y)=0$ if, and only if, $f(y, x)=0$, we see that $f(x, y)$ is actually a skew-symmetric bilinear form.

It is well known ${ }^{4}$ that there exists to every skew-symmetric, $F$ valued bilinear form $f(x, y)$ over $(F, n)$ a basis $x(1), \cdots, x(m)$, $y(1), \cdots, y(m), z(1), \cdots, z(k)$ of $(F, n)$, meeting the following requirements:

$$
0 \leqq m, \quad 0 \leqq k, \quad 2 m+k=n+1 ;
$$

(b) $f(x(i), y(i))=1$ for $1 \leqq i \leqq m$;

$$
f(x(i), x(j))=f(y(i), y(j))=0 \text { for every } i \text { and } j \text {; }
$$

(c) $\quad f(x(i), z(j))=f(y(i), z(j))=f(z(i), z(j))=0$ for every $i$ and $j$; $f(x(i), y(j))=0$ for every $i \neq j$.

This implies in particular $f(x, z(i))=0$ for every $x$ in $(F, n)$ so that the hyperplane $(F z(i))^{p}$ would contain every point of the space, an impossibility proving $k=0$ and $n=2 m-1$. Summarizing our results we obtain:

If $P$ is a projective space of dimension $n$, greater than 1 , and if $p$ is a null-polarity in $P$, then $n=2 m-1$ for $m$ a positive integer, $P$ is the $n$-dimensional projective space over a commutative field $F$, and there exists a system of homogeneous coordinates in $P$ such that the point $F\left(x_{0}, \cdots, x_{2 m-1}\right)$ is on the hyperplane $\left[F\left(y_{0}, \cdots, y_{2 m-1}\right)\right]^{p}$ if, and only if,

$$
0=\sum_{i=0}^{m-1}\left(x_{2 i+1} y_{2 i}-y_{2 i+1} x_{2 i}\right) .
$$

Combining all our results one deduces without difficulty the following facts.

\footnotetext{
${ }^{4}$ See for instance C. C. MacDuffee, The theory of matrices, Ergebnisse der Mathematik under ihrer Grenzgebiete, vol. 2, part 5, 1933, pp. 52, 53, Theorem 32.2.
} 
TheOREM A. In an n-dimensional projective space there exists essentially at most one null-polarity.

THEOREM B. In the $n$-dimensional projective space $P$ with $1<n$ there exists a null-polarity if, and only if, $n$ is odd and $P$ is the projective space of dimension $n$ over a commutative field.

UNIVERSITY OF ILLINOIS 